\title{
BMJ Open Cross-sectional study to predict subnational levels of health workers' knowledge about severe malaria treatment in Kenya
}

\author{
Beatrice Machini (1) ,1,2 Thomas NO Achia, ${ }^{1,3}$ Jacqueline Chesang, ${ }^{1}$ \\ Beatrice Amboko, ${ }^{4}$ Paul Mwaniki, ${ }^{4}$ Hillary Kipruto ${ }^{5}$
}

To cite: Machini B, Achia TNO, Chesang J, et al. Crosssectional study to predict subnational levels of health workers' knowledge about severe malaria treatment in Kenya. BMJ Open 2022;12:e058511. doi:10.1136/ bmjopen-2021-058511

\section{- Prepublication history and} additional supplemental material for this paper are available online. To view these files, please visit the journal online (http://dx.doi.org/10.1136/ bmjopen-2021-058511)

Received 19 0ctober 2021 Accepted 16 December 2021

\section{Check for updates}

(c) Author(s) (or their employer(s)) 2022. Re-use permitted under CC BY-NC. No commercial re-use. See rights and permissions. Published by BMJ.

${ }^{1}$ Department of Public and Global Health, University of Nairobi, Nairobi, Kenya ${ }^{2}$ Ministry of Health, Division of National Malaria Programme, Nairobi, Kenya

${ }^{3}$ School of Mathematics and Computer Science, University of Kwa Zulu Natal, Kwa Zulu Natal, South Africa

${ }^{4}$ Health Services Unit, KEMRI - Wellcome Trust Research Institute, Nairobi, Kenya

${ }^{5}$ World Health Organization, Regional Office for Africa, Eastern and Southern Africa, Harare, Zimbabwe

Correspondence to Dr Beatrice Machini; bkemunto2002@gmail.com

\section{ABSTRACT}

Objectives This study applied a Bayesian hierarchical ecological spatial model beyond predictor analysis to test for the best fitting spatial effects model to predict subnational levels of health workers' knowledge of severe malaria treatment policy, artesunate dosing, and preparation.

Setting County referral government and major faith-based hospitals across 47 counties in Kenya in 2019.

Design and participants A secondary analysis of crosssectional survey data from 345 health workers across 89 hospitals with inpatient departments who were randomly selected and interviewed.

Outcome measures Three ordinal outcome variables for severe malaria treatment policy, artesunate dose and preparation were considered, while 12 individual and contextual predictors were included in the spatial models. Results A third of the health workers had high knowledge levels on artesunate treatment policy; almost threequarters had high knowledge levels on artesunate dosing and preparation. The likelihood of having high knowledge on severe malaria treatment policy was lower among nurses relative to clinicians (adjusted $\mathrm{OR}(\mathrm{aOR})=0.48$, $95 \% \mathrm{Cl} 0.25$ to 0.87 ), health workers older than 30 years were $61 \%$ less likely to have high knowledge about dosing compared with younger health workers $(\mathrm{aOR}=0.39$, $95 \% \mathrm{Cl} 0.22$ to 0.67 ), while health workers exposed to artesunate posters had 2.4-fold higher odds of higher knowledge about dosing compared with non-exposed health workers ( $\mathrm{aOR}=2.38,95 \% \mathrm{Cl} 1.22$ to 4.74 ). The best model fitted with spatially structured random effects and spatial variations of the knowledge level across the 47 counties exhibited neighbourhood influence.

Conclusions Knowledge of severe malaria treatment policies is not adequately and optimally available among health workers across Kenya. The factors associated with the health workers' level of knowledge were cadre, age and exposure to artesunate posters. The spatial maps provided subnational estimates of knowledge levels for focused interventions.

\section{INTRODUCTION}

Malaria is a major public health problem. In 2019, there were an estimated 229 million cases of malaria and 409000 deaths due to malaria globally. The WHO African Region
Strengths and limitations of this study

- This study provided insights about health workers knowledge levels and predictors influencing knowledge at subnational levels for focused interventions.

- The Bayesian modelling provided a robust methodology that combined multiple sources of information in a principled way to make reliable inferences.

- Due to multiple exploratory data analyses and comparisons, some of the results may have been significant by chance.

- The knowledge levels were self-reported by health workers working at the inpatient departments in the sampled hospitals and, therefore, should be generalised with caution.

- The study determined the health workers' level of knowledge about artesunate treatment but not their actual practice; hence, the results cannot be used to make inferences about actual clinical practice.

accounted for $94 \%$ of the malaria cases. ${ }^{1}$ In 2020 , the national malaria prevalence rate was $6 \%$ in Kenya. The prevalence varied across the five epidemiological zones, ranging from $19 \%$ in the Lake endemic zone to $0.4 \%$ in the low-risk malaria areas. ${ }^{2}$

Severe malaria is a medical emergency that requires prompt treatment, as it is associated with a high risk of death within the first 24 hours. ${ }^{3}$ In 2012, the WHO recommended the use of parenteral artesunate for the treatment of severe malaria. ${ }^{4}$ This treatment policy has been adopted and implemented across malaria-endemic countries in Africa. ${ }^{3}$ Health workers' knowledge of evidence-based treatment recommendations is one of the basic requirements for a healthcare system's readiness to implement any new drug policy. ${ }^{5}$ In Kenya, concerted efforts have been made to support the WHO policy and monitor its implementation using various health facility surveys that report national levels, trends and predictors of artesunate 
knowledge deficiencies among hospital health workers. ${ }^{56}$ Similar cross-sectional studies have reported inadequate health workers' knowledge of artesunate-based treatment recommendations. ${ }^{78}$ The data from these studies were multilevel and spatially correlated in nature. Traditionally, such data have been analysed by applying cluster adjustments and correlation matrices based on theoretical assumptions, ${ }^{5}$ 6-11 without considering spatial correlations between clusters. ${ }^{12-14}$ Bayesian hierarchical spatial modelling accounts for correlation by introducing effects at different levels of a hierarchy to estimate random effects together with other model parameters accounting for variability within and between sites. ${ }^{15-20}$ The random effects incorporated into fixed-effect models capture the heterogeneity across clusters in the regression coefficients, accounting for the dependence of observations from the same cluster, ${ }^{21-24}$ leading to accurate conclusions. ${ }^{25} 26$ The Bayesian multilevel models account for the spatial heterogeneity existing among groups, and the conditional autoregressive (CAR) models spatial autocorrelation based on neighbourhood relationships. ${ }^{27-32}$ In this study, neighbourhood was defined using queen adjacency, where a county was considered a neighbour if it shared either a vertex or a node. In this study, a Bayesian hierarchical ecological spatial model beyond predictor analysis was applied to test for the best fitting model to predict subnational artesunate knowledge levels across 47 counties in Kenya.

\section{METHODS}

\section{National standards and implementation context}

Since 2012, the severe malaria treatment policy in Kenya recommends that all children and adults, including pregnant women in all trimesters, should be treated with parenteral artesunate. $^{3433}$ Children weighing less than $20 \mathrm{~kg}$ should be given $3.0 \mathrm{mg} / \mathrm{kg}$ per dose of artesunate, while children weighing more than $20 \mathrm{~kg}$ and adults should receive $2.4 \mathrm{mg} /$ $\mathrm{kg}$ of artesunate. Artesunate is dispensed as a powder of artesunic acid, which is dissolved in sodium bicarbonate $(5 \%)$ to form sodium artesunate. The solution is then diluted using normal saline ( $0.9 \%$ sodium chloride) or $5 \%$ dextrose solution to make a concentration suitable for either intravenous or intramuscular administration. The supportive activities for implementing the treatment policy included a nationwide supply of artesunate, revision and dissemination of malaria treatment guidelines and job aids, and in-service training of front-line health workers on broader malaria case management with more in-depth information on the use and effectiveness of artesunate in managing severe malaria. ${ }^{6}$

\section{Data sources}

The manuscript presents a secondary data analysis of the cross-sectional cluster sample survey undertaken in 2019 to monitor the progress of the health systems' readiness and the quality of inpatient malaria management in Kenyan hospitals. The survey was undertaken at all 47 counties in Kenya, including a major government and

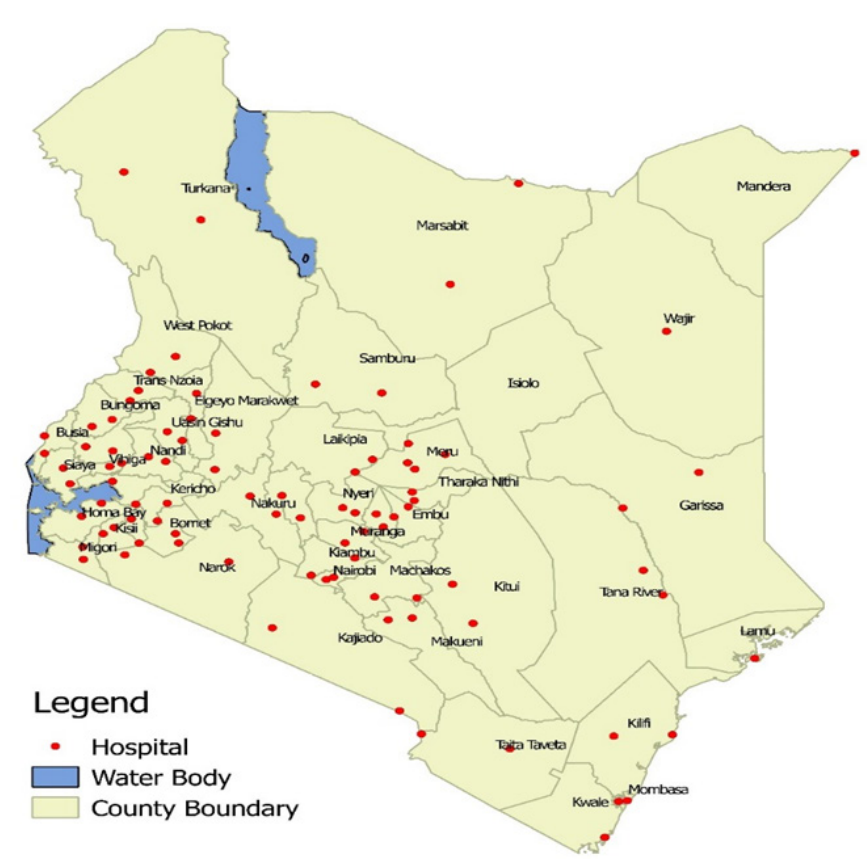

Figure 1 Map of survey hospitals.

faith-based hospital in every county. In each hospital, a randomly sampled clinician and nurse on duty in specific paediatric and medical wards of interest was surveyed (figure 1). Details of the methodologies have been previously published. ${ }^{5}$ Of relevance for this paper, the interviews with health workers were undertaken by trained and experienced study nurses to establish demographic characteristics of the respondents, their exposure to supportive interventions, and knowledge about artesunate treatment using a self-administered multiple-choice questionnaire. The availability of medicines and job aids was assessed in hospital pharmacy and admission wards of interest.

\section{Patient and public involvement statement}

Patients and public were not involved in the design, execution, reporting or dissemination of this research.

\section{Outcomes, definitions and factors examined}

The study considered three response variables reflecting the correctness of the health workers' knowledge about recommended antimalarial treatment for severe malaria, artesunate dose and preparation. These variables were constructed using a multiple correspondence analysis (MCA) approach based on variables measured during the survey. MCA is a data analysis technique for nominal categorical data and is used to detect and represent underlying structures in a complex dataset. ${ }^{34}$ Prior to computing the MCA, the health worker outcome variables were recoded into dichotomous variables, allowing the variables to take a value of zero or one. The resulting polytomous knowledge response was ordered on a three-point scale: high, medium or low. The definitions of the knowledge categories for each outcome assessed are listed in table 1 . 
Table 1 Categories of knowledge outcomes, national standards and study definitions

\begin{tabular}{|c|c|c|c|}
\hline $\begin{array}{l}\text { Knowledge } \\
\text { outcomes }\end{array}$ & National recommendations & $\begin{array}{l}\text { Knowledge } \\
\text { categories }\end{array}$ & Category definitions \\
\hline \multirow[t]{3}{*}{$\begin{array}{l}\text { Treatment policy for } \\
\text { severe malaria }\end{array}$} & \multirow{3}{*}{$\begin{array}{l}\text { Artesunate for the following three severe } \\
\text { malaria populations: } \\
\text { 1. children and non-pregnant adults; } \\
\text { 2. pregnant women in first trimester; } \\
\text { 3. pregnant women in second \& third } \\
\text { trimesters }\end{array}$} & High & $\begin{array}{l}\text { Artesunate response for all three severe } \\
\text { malaria populations }\end{array}$ \\
\hline & & Medium & $\begin{array}{l}\text { Artesunate response for two severe } \\
\text { malaria populations }\end{array}$ \\
\hline & & Low & $\begin{array}{l}\text { Artesunate response for one or none of } \\
\text { the populations }\end{array}$ \\
\hline \multirow[t]{3}{*}{ Artesunate dose } & \multirow{3}{*}{$\begin{array}{l}2 \text { wt categories: } \\
-3 \mathrm{mg} / \mathrm{kg} \text { for child }<20 \mathrm{~kg}, \\
>2.4 \mathrm{mg} / \mathrm{kg} \text { for patient }>20 \mathrm{~kg}\end{array}$} & High & Correct response for 2 wt categories \\
\hline & & Medium & Correct response for one weight category \\
\hline & & Low & $\begin{array}{l}\text { Incorrect responses for all the weight } \\
\text { categories }\end{array}$ \\
\hline $\begin{array}{l}\text { Artesunate } \\
\text { preparation }\end{array}$ & $\begin{array}{l}\text { Solutions for two artesunate preparation } \\
\text { steps: } \\
\text { 1. bicarbonate for reconstitution } \\
\text { 2. saline or } 5 \% \text { dextrose for dilution }\end{array}$ & High & $\begin{array}{l}\text { Correct response for two preparation } \\
\text { steps }\end{array}$ \\
\hline
\end{tabular}

The variables selected for analysis were based on previous studies ${ }^{5}$ and classified as individual or contextual predictors. The health worker-level attributes were considered as individual predictors and they included: gender (male vs female), cadre (clinician vs nurse), age (21-30 vs 31-60), years of experience ( $<10$ years vs $>10$ years), admission ward allocation (medical vs paediatric), artesunate training (yes vs no), access to malaria guidelines (yes vs no), and access to paediatric protocols (yes vs no). The contextual variables presented heath facility level characteristics, including availability of artesunate (yes vs no), display of artesunate administration posters (yes vs no), availability of artesunate dosing job aids (yes vs no) and malaria endemicity classification (high vs low).

\section{Statistical analysis}

\section{Summary and exploratory analysis}

Descriptive statistics were used to summarise the demographic characteristics of health workers and health facilities. Subsequently, applying the Bayesian approach, univariate analysis, estimated OR and credible intervals (CI) were calculated. Significant predictors (80\% CI) associated with the level of health workers' knowledge of severe malaria treatment policy, artesunate dosing and preparation were identified and included in the multilevel modelling. Then, an ordinal logistic regression analysis adjusted for clustering at the county level was performed to estimate the effects of the predictor variable on the response variable $(95 \%$ CI) by fitting three hierarchical models.

\section{Bayesian method for ordinal logistic regression model}

The ordinal logit model was developed from the general form of the binomial models. The model can be expressed as a latent variable model, ${ }^{35}$ a powerful class of models for treating observations that fall into mutually exclusive categorical classes. The flexibility of this regression framework allows for better inferences. Initially, ordinal logistic regression analysis was implemented, followed by Bayesian hierarchical spatial modelling, as shown below:

Let $Y_{i j}$ be a trichotomous outcome variable taking values 1,2 , or 3 if the $j$ th health worker in the $i$ th county $i=1, \ldots, 47$ had low, medium or high artesunate knowledge, respectively.

In this study, three versions of the cumulative link model for ordinal-scale observations were fitted as follows:

$$
\begin{aligned}
\log \left(\frac{\gamma_{i j k}}{1-\gamma_{i j k}}\right)= & \theta_{k}-\left(x_{i j}^{T} \beta+u_{i}+v_{i}\right), \\
& i=1, \ldots, 47, j=1, \ldots, n_{i}, k=1,2,3,
\end{aligned}
$$

where

$$
\begin{aligned}
\gamma_{i j k}= & P\left(Y_{i j} \leq k\right)=\pi_{i j 1}+\pi_{i j 2}+ \\
& \ldots+\pi_{i j k} \text { with } \sum_{k=1}^{3} \pi_{i j k}=1
\end{aligned}
$$

are cumulative probabilities, $x_{i j}^{T}$ is a $p$-vector of regression variables for the parameters, $\beta$ without a leading column for an intercept; $\theta_{k}, k=0,1,2,3$ are thresholds for the cumulative ordinal logit model; $u_{i}$ is a spatial structured component random effect for the $i$-th county with a CAR distribution $u_{i} \mid \boldsymbol{u}_{-i} \sim N\left(\bar{u}_{\delta_{i}}, \frac{\sigma_{u}^{2}}{n_{\delta_{i}}}\right)$, where $\bar{u}_{\delta_{i}}=n_{\delta_{i}}^{-1} \sum_{j \in \delta_{i}} u_{j} \delta_{i}$ and $n_{\delta_{i}}$ represent the set of neighbours and the number of neighbours for the $i$-th county, respectively; and $v_{i}$ is an unstructured spatial random effect for the $i$-th county defined as $v_{i} \sim N\left(0, \sigma_{v}^{2}\right)$. The first model and second models (Model 1, Model 2) were ordinal logistic regressions with spatially structured and unstructured random effects respectively, the third model (Model 3) was a convolution model fit by combining both structured and unstructured spatial random effects. Online supplemental information provides a more detailed description of the Bayesian hierarchical spatial modelling. 


\section{Bayesian statistical inference}

During the model assessment, significant individual and contextual predictors were included in the model simultaneously. The predictive performance of the three hierarchical models was compared using the deviance information criterion (DIC), and a smaller DIC was regarded as a better model. Sensitivity analysis was performed by assuming three chains of Markov Chain Monte Carlo (MCMC) algorithms, ${ }^{37}$ specifying the same model and prior information from different starting values and comparing the variance within each chain with the variance between chains. Large MCMC samples were used to establish better estimates. In executing this analysis, 10000 iterations with a burn-in of 500 and thinning of one were run to reduce autocorrelation and avoid bias in the SE estimate of the posterior mean. Model convergence was assessed using trace plots, histograms and autocorrelation graphs, monitored by R-hat convergence diagnostic, which is the ratio of the spread of all the values combined with the mean spread of each chain. The posterior means/OR, quantiles, median, SD and the corresponding $95 \%$ CI were used to assess the significance of all parameters. ${ }^{38}$ The spatial random effects from the best fitting model (structured, unstructured or convolution) of health workers with high knowledge of treatment policy, artesunate dosing and preparation were overlaid on a map showing all counties in Kenya. Initial analysis was conducted using StataCorp V.14 (Stata Statistical Software: Release 14. College Station, TX, StataCorp LP). The Bayesian models were fitted using the R2OpenBUGS statistical package.

\section{RESULTS}

\section{Health worker characteristics}

Of the 345 interviewed health workers, most were female $(59.7 \%)$, aged $21-30$ years $(62 \%)$, with less than 10 years of inpatient experience $(82.6 \%)$, and working in low malaria risk areas $(72.5 \%)$. A quarter of them $(24.6 \%)$ had access to dosing job aids, $36.8 \%$ had been trained on artesunate use and $40.9 \%$ had access to malaria treatment guidelines. Most of the health workers worked at hospitals with artesunate in stock $(90.7 \%)$ and displayed artesunate administration posters $(82.9 \%)$. The health worker ward allocation, cadre and paediatric protocol exposures were similarly distributed in the sample (table 2).

\section{Health workers level of knowledge on severe malaria treatment policy, artesunate dose and preparation}

The reliability of the indices constructed by the MCA approach was assessed using Cronbach's alpha coefficient and a score of $>0.7$ indicated high intracorrelation among a set of variables. ${ }^{34}$ The Cronbach alpha coefficients for knowledge about severe malaria treatment policy, artesunate dosing and preparation were $0.7674,0.8901$ and 0.7810 , respectively. The resulting polytomous knowledge response was ordered as high, medium, or low.
Table 2 Distribution of the health workers' characteristics

\begin{tabular}{|c|c|c|}
\hline & \multicolumn{2}{|c|}{$\mathrm{N}=345$} \\
\hline & $\mathrm{n}$ & Per cent (\%) \\
\hline \multicolumn{3}{|l|}{ Predictor variables } \\
\hline \multicolumn{3}{|l|}{ Gender } \\
\hline Male & 139 & 40.3 \\
\hline Female & 206 & 59.7 \\
\hline \multicolumn{3}{|l|}{ Health worker cadre } \\
\hline Clinician & 159 & 46.1 \\
\hline Nurse & 186 & 53.9 \\
\hline \multicolumn{3}{|l|}{ Age } \\
\hline $21-30$ & 214 & 62.0 \\
\hline $31-60$ & 131 & 38.0 \\
\hline \multicolumn{3}{|l|}{ Years of experience } \\
\hline$>10$ years & 60 & 17.4 \\
\hline$<10$ years & 285 & 82.6 \\
\hline \multicolumn{3}{|l|}{ Ward allocation } \\
\hline Medical & 170 & 49.3 \\
\hline Paediatric & 175 & 50.7 \\
\hline \multicolumn{3}{|c|}{ Exposure to artesunate interventions } \\
\hline Trained on artesunate & 127 & 36.8 \\
\hline Malaria treatment guidelines & 141 & 40.9 \\
\hline Paediatric protocol & 186 & 53.9 \\
\hline Artesunate poster & 286 & 82.9 \\
\hline Artesunate dosing wheel & 85 & 24.6 \\
\hline Availability of artesunate & 313 & 90.7 \\
\hline \multicolumn{3}{|l|}{ Endemicity } \\
\hline Low & 250 & 72.5 \\
\hline High & 95 & 27.5 \\
\hline
\end{tabular}

A third of the health workers had a high level of knowledge about artesunate treatment policy for severe malaria $(32.8 \%)$, while $73.9 \%$ and $70.9 \%$ of health workers had high levels of knowledge about the recommended artesunate dosing and preparation, respectively (table 3 ). Online supplemental tables 1-3 show the results of univariate ordinal logistic regression analyses examining the association between 12 factors and three knowledge outcomes. Of the 12 factors examined, 2, 10 and 1 factor(s) met the inclusion criteria for multivariable analysis $(80 \% \mathrm{CI})$ with knowledge about treatment policy, artesunate dosing and artesunate preparation, respectively.

Table 4 reports the results of the three comparative hierarchical models that were fitted in multivariable analysis and their goodness of fit is compared using DIC. For the health workers' knowledge on treatment policy, the DIC for model 1 , model 2 and model 3 were 762.71, 780.17 and 770.14, respectively. Regarding health workers' knowledge on artesunate dosing, the DIC for model 1, model 2 and model 3 were 488.83, 496.19 and 497.80, respectively. For the health workers' knowledge 
Table 3 Knowledge levels about artesunate treatment

\section{Distribution of outcome variables}

\begin{tabular}{lrc}
\hline & \multicolumn{2}{l}{$\mathbf{N = 3 4 5}$} \\
\cline { 2 - 3 } Knowledge categories & $\mathbf{n}$ & Per cent (\%) \\
\hline Treatment policy & & \\
High & 113 & 32.8 \\
\hline Medium & 107 & 31.0 \\
\hline Low & 125 & 36.2 \\
\hline Dosing & & \\
High & 255 & 73.9 \\
\hline Medium & 57 & 16.5 \\
\hline Low & 33 & 9.6 \\
\hline Artesunate preparation ${ }^{\star}$ & & \\
\hline High & 244 & 70.9 \\
\hline Medium & 85 & 24.7 \\
\hline Low & 15 & 4.4 \\
\hline
\end{tabular}

*has one missing value.

on artesunate preparation, the DIC for model 1, model 2 and model 3 were 503.31, 510.17 and 507.31, respectively. Model 1 with spatially structured random effects provided a better fit for the three outcomes. Spatially structured random effects illustrate the necessity of accounting for spatial autocorrelation, which, if ignored in the regression model, can lead to biased inferences.

The posterior means/OR, quantiles, median, SD and the corresponding $95 \%$ CI were used to assess the significance of all parameters. The posterior estimates were similar across the three hierarchical models and the adjusted ORs and 95\% CI estimates from the best fitting model are reported. For the outcome on the knowledge about artesunate treatment policy, the health workers' cadre was the only significant predictor. The likelihood of having a high knowledge of severe malaria treatment policy was significantly lower in nurses than in clinicians (aOR $=0.59,95 \%$ CI 0.40 to 0.89 ). Regarding knowledge of the recommended artesunate dosing, health worker cadre, age and exposure to artesunate administration poster were significant predictors. Nurses were $52 \%$ less likely to have high knowledge about dosing compared with the clinicians $(\mathrm{aOR}=0.48,95 \%$ CI 0.25 to 0.87$)$. Health workers older than 30 years were $61 \%$ less likely to have high knowledge about dosing compared with younger health workers ( $\mathrm{aOR}=0.39,95 \% \mathrm{CI} 0.22$ to 0.67 ), while health workers exposed to artesunate posters had 2.4-fold increased odds of higher knowledge about dosing compared with non-exposed health workers $(\mathrm{aOR}=2.38$, 95\% CI 1.22 to 4.74). Finally, based on unadjusted univariate analysis (online supplemental table 3), the health workers who had access to an artesunate dosing wheel were $57 \%$ more likely to have higher knowledge of artesunate preparation compared with those who did not have access $(\mathrm{OR}=1.57,80 \%$ CI 1.09 to 2.30$)$. However, the same predictor variable lost significance at the $95 \%$
CI, adjusted for multivariable analysis $(\mathrm{aOR}=1.58,95 \% \mathrm{CI}$ 0.91 to 2.88), refer to online supplemental table 4 .

Model 1: spatially structured random effects; model 2: spatially unstructured random effects; model 3 : convolution Figures 2-4 show the spatial random effects of the posterior means of the probability of health workers having high knowledge of severe malaria treatment policy, artesunate dosing and preparation, respectively, overlaid on a map showing all counties in Kenya. The deep red colour denotes regions with strictly high knowledge, while the light red colour denotes strictly low knowledge. In figure 2, the health workers in Kisii county had high knowledge levels $(>10 \%)$ on severe malaria treatment policy, while those in Nyandarua, Nyamira, Laikipia and Mandera counties had low knowledge levels $(<10 \%)$. In figure 3, the health workers in Muranga, Kisii, Embu, Uasin Gishu, Kiambu and Kisumu counties had high knowledge levels $(>10 \%)$ about artesunate doses, while those in Nyandarua, Nyamira, Garissa, Busia and Nairobi counties had low knowledge levels $(<10 \%)$. In figure 4 , there were 17 counties with high knowledge levels $(>10 \%)$, while 16 counties had low knowledge levels $(<10 \%)$, on artesunate preparation.

\section{DISCUSSION}

This study applied Bayesian hierarchical ecological spatial modelling as an extension to the standard approach to examine the spatial effects at the national level on Kenyan health workers' knowledge of severe malaria treatment policy, artesunate dosing and preparation. Three ordinal response variables for severe malaria treatment policy, artesunate dose and preparation were considered, while 12 individual and contextual predictors were included in the models. The analysis was performed using three different models: the first model was ordinal logistic regression with spatially structured random effects, the second model with spatially unstructured random effects and the third with convolution. This Bayesian approach provided another way of examining factors associated with health workers' level of knowledge and the spatial factors around severe malaria treatment policies for targeted malaria interventions.

Knowledge required to treat severe malaria is not adequately and optimally spread among all health workers at the national level. A third of the health workers had high knowledge levels about WHO's artesunate treatment policy. Previous studies have reported similar findings. 568 This was linked to the low knowledge levels on the treatment policy for pregnant women in the first and second trimesters. Almost three-quarters of health workers had high knowledge levels on the correct artesunate dosing recommendations for patients weighing both below and above $20 \mathrm{~kg}$ and were aware of both artesunate preparation solutions. The majority of health workers have not been trained on artesunate treatment policy. There is a need to organise more training avenues, such as seminars 


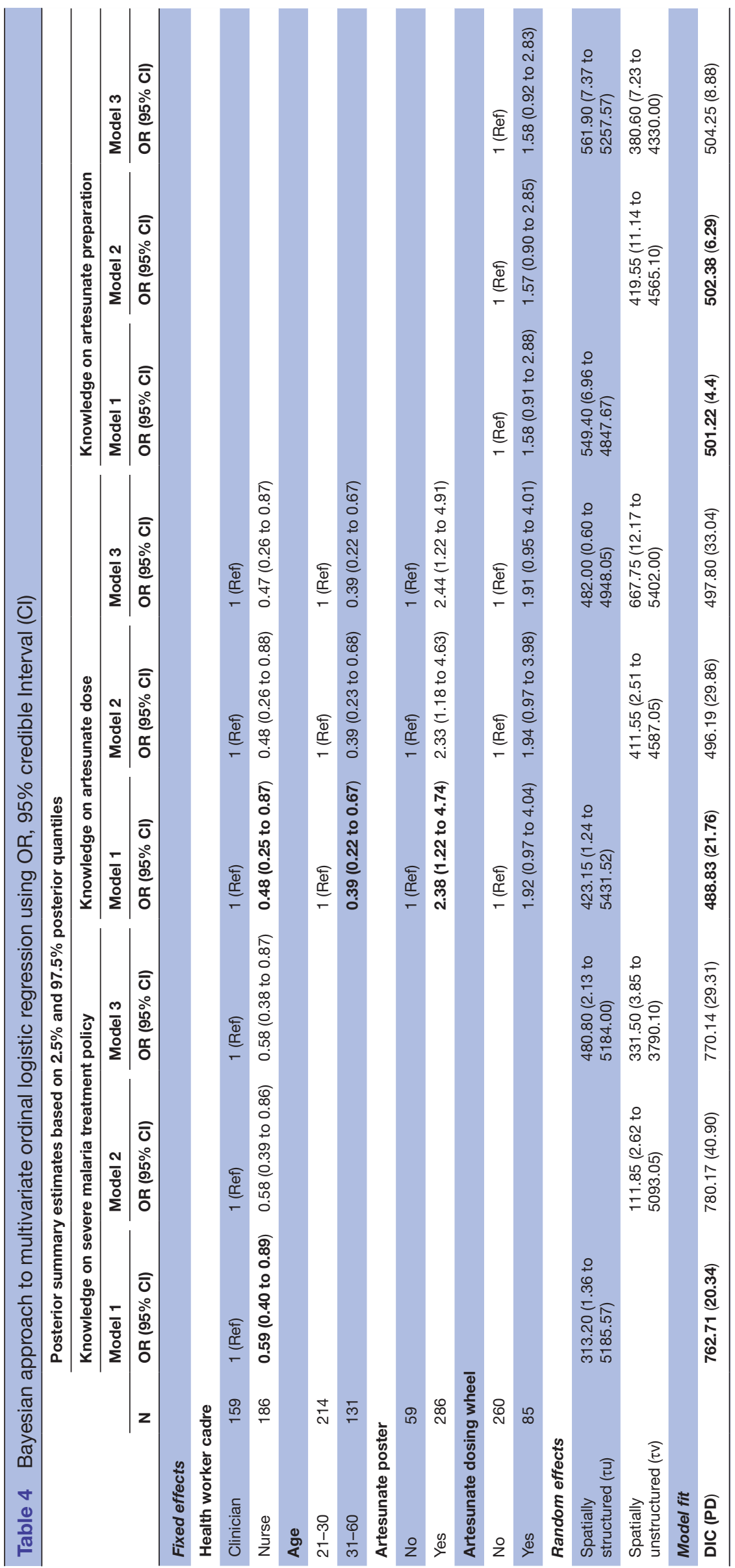

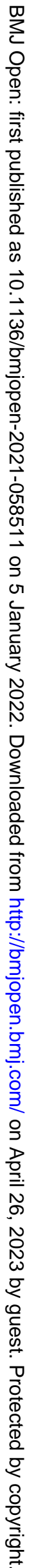




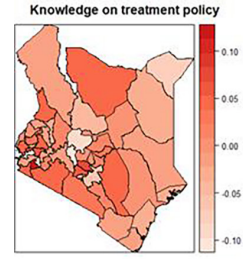

A Posterior mean

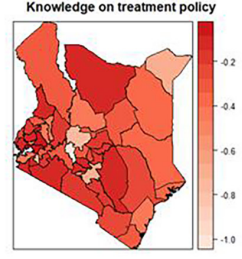

B $2.5 \%$ quandiles of posterior mean

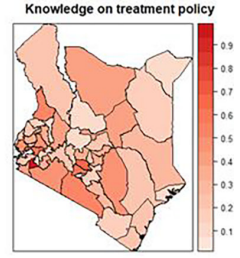

C $97.5 \%$ quandiles of posterior maan
Figure 2 Spatially structured random effects on probability of health workers having high knowledge on the recommended treatment policy of severe malaria using artesunate. (A) Posterior mean, (B) 2.5\% quantiles and (C) $97.5 \%$ quantiles.

and workshops, and to re-evaluate the mode of delivery of training conducted in the past.

Nurses were less likely than clinicians to have a high level of knowledge of the WHO treatment policy for severe malaria, consistent with other research findings. ${ }^{8}{ }^{89}$ There is a need for interprofessional collaboration and innovative measures to improve knowledge of the severe malaria treatment policy and dosing among nurses in future case management training. The health workers above 30 years of age had low knowledge of artesunate dosing. These health workers are a critical segment of the workforce and should be targeted for refresher training on severe malaria case management. It is commendable that the artesunate poster contributes to health workers' level of knowledge of artesunate dosing. A similar observation was made in Tanzania that healthcare workers relied on posters to prepare injectable artesunate. ${ }^{8}$ The programme should continue updating, printing and disseminating more posters to all health facilities to supplement the knowledge gained through training.

There is evidence of spatially structured variation in health workers' knowledge of severe malaria treatment policy, artesunate dosing and preparation at various county hospitals. The best fitting model for severe malaria treatment policy, artesunate dosing and preparation was fitted with spatially structured random effects. The similarity of responses from health workers interviewed in a given facility and the likelihood of similarity between health facility structures across adjacent counties explain the neighbourhood influence on the spatially structured models. The substantial heterogeneity among the health workers with high knowledge of treatment policy,

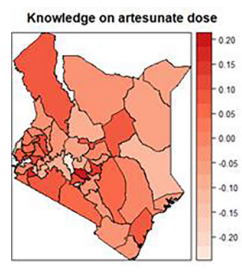

A Posterior man

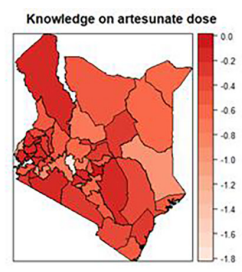

B $2.5 \%$ quantiles of psterior mata

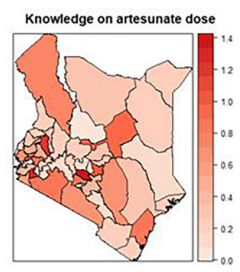

C $97.5 \%$ quandiles of posterior mann
Figure 3 Spatially structured random effects on probability of health workers having high knowledge on artesunate dose. (A) Posterior mean, (B) 2.5\% quantiles, and (C) $97.5 \%$ quantiles.

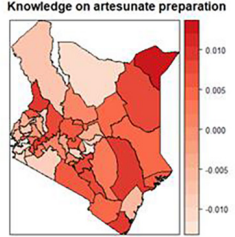

A Posterior mean

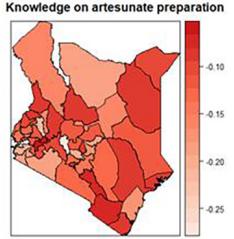

B $2.5 \%$ quantiles of posterior mean

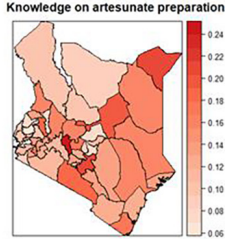

C $97.5 \%$ quantiles of posterior mean
Figure 4 Spatially structured random effects on probability of health workers having high knowledge on artesunate preparation. (A) Posterior mean, (B) 2.5\% quantile and (C) $97.5 \%$ quantile.

artesunate dosing and preparation at the county level showed that severe malaria management may differ from one health facility or county to another due to unobserved heterogeneity, and this required Bayesian hierarchical model to account for clustering within health facilities and counties. ${ }^{40}$ Bayesian hierarchical spatial models account for both the nesting of health workers within health facilities (vertical dependence) and the spatial autocorrelation among the counties (horizontal dependence) by assigning a normal CAR prior to the random effects. ${ }^{41}{ }^{42}$ Bayesian spatial models incorporate geographical correlation by a CAR prior to assess small area variations and map spatial patterns. ${ }^{43}$ The models assume that geographically proximate spatial units tend to have similar risks, illustrating the necessity of accounting for spatial variation across the counties for precise inferences. The spatially unstructured random variables ignore the geographical location of the analysis units by capturing the unobserved non-spatial heterogeneity. ${ }^{44-46}$ The spatial maps provide subnational knowledge level estimates that can be used for focused interventions.

\section{Strengths and limitations}

This study provided insights into health workers' knowledge levels and predictors influencing knowledge at subnational levels for focused interventions. Bayesian modelling provided a robust methodology that combined multiple sources of information in a principled way to perform reliable inferences. This study has a few limitations. First, due to multiple exploratory data analyses and comparisons, some of the results may have been significant by chance. Second, the knowledge levels were self-reported by health workers working in the inpatient departments at the sampled hospitals and should be generalised with caution. Third, the study determined the health workers' level of knowledge on artesunate treatment but not their actual practice; hence, the results cannot be extended to infer about actual clinical practice.

\section{CONCLUSION}

The individual factors associated with health workers' knowledge of severe malaria treatment policy, dosing and preparation were health worker cadre and age, while exposure to artesunate posters was a contextual factor. At the health facility level, various targeted strategies such as 
job training, continuous medical education with a multidisciplinary approach, and case management training with emphasis on dosing should be explored to improve the knowledge base of health workers for severe malaria management. Continued routine dissemination of information and display of artesunate posters to health facilities is encouraged.

Based on the spatial maps, the National Malaria Programme can focus interventions with a multidisciplinary approach to bridge the knowledge gaps identified at the subnational level. Bayesian methodology can be adopted to analyse health survey data with similar structures and settings. A qualitative study is recommended to uncover why the current operational interventions have not improved health workers' knowledge of the severe malaria treatment policy.

Acknowledgements The authors would like to thank the Division of National Malaria Programme and the County Health Management Teams for their support in this study. We thank the hospital nurses and clinicians who participated in this study. We are also grateful to Peter Macharia and Dejan Zurovac for comments on an earlier version of this manuscript. We would like to thank Editage (www.editage. com) for English language editing.

Contributors BM, TNOA and HK designed the study. JC, PM and BA contributed to the analysis and interpretation of the study findings. BM wrote the first draft of this manuscript. All authors reviewed and approved the final version of the manuscript. $\mathrm{BM}$ is the author acting as guarantor.

Funding Funding for malaria case-management surveys is provided by the Global Fund to Fight AIDS, Tuberculosis, and Malaria, Grant. BM is not supported, and the views expressed in this publication are those of the author(s).

Map disclaimer The depiction of boundaries on this map does not imply the expression of any opinion whatsoever on the part of BMJ (or any member of its group) concerning the legal status of any country, territory, jurisdiction or area or of its authorities. This map is provided without any warranty of any kind, either express or implied.

\section{Competing interests None declared.}

Patient consent for publication Not applicable.

Ethics approval This study involves human participants and was approved by Kenyatta National Hospital/University of Nairobi-Ethics Committee (KNH/UON/ERC/ P233/04/2018). Participants gave informed consent to participate in the study before taking part.

Provenance and peer review Not commissioned; externally peer reviewed.

Data availability statement Data are available upon reasonable request. The datasets used and analysed during the current study are available from the corresponding author on reasonable request.

Supplemental material This content has been supplied by the author(s). It has not been vetted by BMJ Publishing Group Limited (BMJ) and may not have been peer-reviewed. Any opinions or recommendations discussed are solely those of the author(s) and are not endorsed by BMJ. BMJ disclaims all liability and responsibility arising from any reliance placed on the content. Where the content includes any translated material, BMJ does not warrant the accuracy and reliability of the translations (including but not limited to local regulations, clinical guidelines, terminology, drug names and drug dosages), and is not responsible for any error and/or omissions arising from translation and adaptation or otherwise.

Open access This is an open access article distributed in accordance with the Creative Commons Attribution Non Commercial (CC BY-NC 4.0) license, which permits others to distribute, remix, adapt, build upon this work non-commercially, and license their derivative works on different terms, provided the original work is properly cited, appropriate credit is given, any changes made indicated, and the use is non-commercial. See: http://creativecommons.org/licenses/by-nc/4.0/.

\section{ORCID iD}

Beatrice Machini http://orcid.org/0000-0001-6457-6541
REFERENCES

1 World Health Organization. World malaria report. World Health Organization, 2020, 2019.

2 Division of national malaria programme (dNMP), Kenya national Bureau of statistics (KNBS), ICF international. Kenya malaria indicator survey 2020. Rockville; Nairobi, 2021.

3 Ministry of Health. National guidelines for diagnosis, treatment and prevention of malaria for health workers in Kenya. Nairobi, Kenya: Division of National Malaria Programme, 2015.

4 World Health Organization. Guidelines for the treatment of malaria. World Health Organization, 2015.

5 Machini B, Zurovac D, Amboko B, et al. Predictors of health workers knowledge about artesunate-based severe malaria treatment recommendations in government and faith-based hospitals in Kenya. Malar J 2020;19:267

6 Zurovac D, Machini B, Kiptui R, et al. Monitoring health systems readiness and inpatient malaria case-management at Kenyan County hospitals. Malar J 2018;17:213.

7 Ojo AA, Maxwell K, Oresanya O, et al. Health systems readiness and quality of inpatient malaria case-management in Kano state, Nigeria. Malar J 2020;19:384.

8 Mikomangwa WP, Kaaya C, Kilonzi M, et al. Level of knowledge among health care providers on preparation of injectable artesunate for treatment of severe malaria in public health facilities in Tanzania. BMC Res Notes 2019;12:224.

9 Zurovac D, Githinji S, Memusi D, et al. Major improvements in the quality of malaria case-management under the "test and treat" policy in Kenya. PLoS One 2014;9:e92782.

10 Amboko B, Stepniewska K, Macharia PM, et al. Trends in health workers' compliance with outpatient malaria case-management guidelines across malaria epidemiological zones in Kenya, 20102016. Malar J 2020;19:406.

11 Moen EL, Fricano-Kugler CJ, Luikart BW, et al. Analyzing clustered data: why and how to account for multiple observations nested within a study participant? PLoS One 2016;11:e0146721.

12 Corani G, Benavoli A, Demšar J, et al. Statistical comparison of classifiers through Bayesian hierarchical modelling. Mach Learn 2017;106:1817-37.

13 Berger JO, De Oliveira V, Sansó B. Objective Bayesian analysis of spatially correlated data. J Am Stat Assoc 2001;96:1361-74.

14 Shor B, Bafumi J, Keele L, et al. A Bayesian multilevel modeling approach to time-series cross-sectional data. Polit. anal. 2007;15:165-81.

15 Box GE, Tiao GC. Bayesian inference in statistical analysis. John Wiley \& Sons, 2011.

16 Kruschke JK, Liddell TM. Bayesian data analysis for newcomers. Psychon Bull Rev 2018;25:155-77.

17 Kruschke JK. Bayesian data analysis. WIREs Cognitive Science 2010;1:658-76.

18 Kruschke JK, Vanpaemel W. Bayesian estimation in hierarchical models. The Oxford Handbook of computational and mathematical psychology, 2015279 Mar 20.

19 Sánchez G, StataCorp LL. Introduction to Bayesian analysis in Stata, 2017.

20 Perezgonzalez JD, Dienes Z. Commentary: how Bayes factors change scientific practice. Front Psychol 2016;7:78-89.

21 Sharifi-Malvajerdi S, Zhu F, Fogarty CB, et al. Malaria parasite clearance rate regression: an $\mathrm{R}$ software package for a Bayesian hierarchical regression model. Malar J 2019;18:4.

$22 \mathrm{Li} \mathrm{W}$, Fearnhead P. Convergence of regression-adjusted approximate Bayesian computation. Biometrika 2018;105:301-18.

23 Dienes Z. Bayesian versus orthodox statistics: which side are you on? Perspect Psychol Sci 2011;6:274-90.

24 Austin PC, Merlo J. Intermediate and advanced topics in multilevel logistic regression analysis. Stat Med 2017;36:3257-77.

25 Bae H, Monti S, Montano M, et al. Learning Bayesian networks from correlated data. Sci Rep 2016;6:25156.

26 Dickinson LM, Basu A. Multilevel modeling and practice-based research. Ann Fam Med 2005;3 Suppl 1:S52-60.

27 Lawson A, Lee D. Bayesian disease mapping for public health. In: Handb STAT. Elsevier, 2017: 36.

28 Obaromi D. Spatial modelling of some conditional autoregressive priors in a disease mapping model: the Bayesian approach. Biomed J Sci Tech Res 2019;14.

29 Aswi A, Cramb S, Duncan E, et al. Evaluating the impact of a small number of areas on spatial estimation. Int $J$ Health Geogr 2020;19:39.

30 Gelman A, Carlin JB, Stern HS. Bayesian data analysis. New York: CRC Press, 2013.

31 Wang $X$, Shao C, Yin C, et al. Exploring the influence of built environment on CAR ownership and use with a spatial multilevel 
model: a case study of Changchun, China. Int J Environ Res Public Health 2018;15:1868.

32 Liu H, Zhu X. Exploring the influence of neighborhood characteristics on burglary risks: a Bayesian random effects modeling approach. ISPRS Int J Geoinf 2016;5:102.

33 Wilairatana P, Tangpukdee N, Krudsood S. Practical aspects of artesunate administration in severe malaria treatment. 10.4172/23299088 2013;1.

34 Ayele D, Zewotir T, Mwambi H. Multiple correspondence analysis as a tool for analysis of large health surveys in African settings. Afr Health Sci 2014;14:1036-45.

35 Agresti A. Categorical data analysis; 482. John Wiley \& Sons, 2003.

36 Tutz G. Regression for categorical data. Cambridge University Press, 2011.

37 Salinelli E, Tomarelli F. Markov chains. discrete dynamical models. Springer, 2014

38 Edwards W, Lindman H, Savage LJ. Bayesian statistical inference for psychological research. Psychol Rev 1963;70:193-242.

39 Reeves S, Pelone F, Harrison R, et al. Interprofessional collaboration to improve professional practice and healthcare outcomes. Cochrane Database Syst Rev 2017;6:CD000072.
40 Hanandita W, Tampubolon G. Geography and social distribution of malaria in Indonesian Papua: a cross-sectional study. Int $J$ Health Geogr 2016;15:13.

41 Wang X, Shao C, Yin C, et al. Application of Bayesian multileve models using small and medium size City in China: the case of Changchun. Sustainability 2018;10:484

42 Umer MF, Zofeen S, Majeed A, et al. Effects of socio-environmental factors on malaria infection in Pakistan: a Bayesian spatial analysis. Int J Environ Res Public Health 2019;16:1365.

43 Dasgupta P, Cramb SM, Aitken JF, et al. Comparing multilevel and Bayesian spatial random effects survival models to assess geographical inequalities in colorectal cancer survival: a case study. Int J Health Geogr 2014;13:36.

44 Mutua MM, Achia TNO, Manderson L, et al. Spatial and socioeconomic correlates of effective contraception among women seeking post-abortion care in healthcare facilities in Kenya. PLOS One 2019;14:e0214049.

45 Rashidi P, Wang T, Skidmore A, et al. Elephant poaching risk assessed using spatial and non-spatial Bayesian models. Ecol Modell 2016;338:60-8.

46 Achia TNO. Spatial modelling and mapping of female genital mutilation in Kenya. BMC Public Health 2014;14:276. 